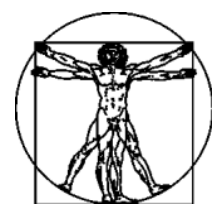

\title{
ОЦЕНКА КОРРЕКТНОСТИ СТАНДАРТНОЙ МЕТОДИКИ РАСЧЕТА ВНУТРИГЛАЗНОГО ДАВЛЕНИЯ ПРИ ТОНОМЕТРИИ ПО ШИОТЦУ
}

\author{
И.Н. Моисеева, А.А. Штейн
}

Институт механики Московского государственного университета им. М.В. Ломоносова, Россия, 119192 , Москва, Мичуринский проспект, 1, e-mail: moiseeva.ir@yandex.ru, stein@imec.msu.ru

\begin{abstract}
Аннотация. Проанализированы зависимости, применяемые при стандартной обработке данных процедуры измерения внутриглазного давления по Шиотцу. В тонометре Шиотца осуществляется совместное нагружение роговицы глаза вогнутой тяжелой стопой и тонким, более легким, вогнутым стержнем (плунжером). Нагружающее устройство имеет с роговицей две зоны контакта - всю вогнутую поверхность стержня и область стопы, определяемую внутренним давлением. При математическом моделировании использована предложенная ранее авторами модель, в которой роговица представлена безмоментной (мягкой) упругой поверхностью, а склеральная область заменяется упругим элементом, откликающимся изменением объема на изменение давления. Стандартная обработка данных тонометрии по Шиотцу базируется на двух эмпирически обоснованных алгоритмах: сначала по измеренному заглублению плунжера под стопу и известному весу плунжера определяется тонометрическое давление, а затем по последнему находится внутриглазное давление в ненагруженном глазу («истинное»). Показано, что использование стандартной методики вполне правомерно для тонометра с минимальным весом стержня 5,5 г, если упругие характеристики глазного яблока близки к средним. При большем весе стержня или значениях упругих констант, сильно отличающихся от нормы, необходимо учитывать поправки. Влияние геометрических параметров роговицы не слишком велико, однако при сильно увеличенном радиусе ее кривизны (например, в результате операции) поправки к результатам стандартного расчета следует учитывать.
\end{abstract}

Ключевые слова: глаз, математические модели, оболочка глаза, внутриглазное давление, тонометрия, тонометр Шиотца.

\section{ВВЕДЕНИЕ}

Прямое измерение внутриглазного давления в условиях стандартного офтальмологического исследования невозможно. Используется неинвазивная измерительная процедура - тонометрия, при выполнении которой давление обычно оценивается по деформациям глазного яблока под нагрузкой, в частности при статической тонометрии - в результате приложения груза к роговице [5]. Основная цель тонометрии - определение истинного внутриглазного давления, т.е. такого давления, которое имело место до измерительного воздействия. Попутно определяется также тонометрическое давление (давление в нагруженном глазу), которое может иметь и самостоятельную диагностическую ценность. При стандартной клинической

(C) Моисеева И.Н., Штейн А.А., 2015

Моисеева Ирина Никитична, к.ф.-м.н., старший научный сотрудник лаборатории общей гидромеханики, Москва

Штейн Александр Александрович, к.ф.-м.н., ведущий научный сотрудник лаборатории общей гидромеханики, Москва 
тонометрии плоским штампом по Маклакову, например, несмотря на существование таблиц пересчета от тонометрического давления к истинному [5], в карту больного вносится именно тонометрическое давление. При тонометрии по Шиотцу, когда роговица испытывает вдавливающее (импрессионное) воздействие, наложенное на напряженное состояние, вызванное воздействием вогнутой тяжелой стопы, разница между тонометрическим и истинным давлением велика и расчет истинного давления (калибровка тонометра) - обязательная составляющая обследования.

Методика расчета истинного давления по показаниям тонометра Шиотца разрабатывалась на основании эмпирических и полуэмпирических исследований в 30-70 гг. прошлого века $[6,8,9]$, и для нее принят международный стандарт. Цель настоящей работы - оценить уровень возможных ошибок при определении истинного и тонометрического внутриглазного давления на основе стандартной методики в зависимости от индивидуальных упругих и геометрических характеристик глазного яблока. Такая оценка необходима в свете нескольких обстоятельств. Известно, что упругие характеристики глаза могут у разных людей различаться на порядок и более [10]. Кроме того, упругие свойства могут по-разному распределяться между роговичной и склеральной областями и изменяться вследствие заболеваний, например глаукомы. А в результате хирургических операций значительно изменяются и геометрические параметры глаза, в первую очередь кривизна роговицы.

В качестве аппарата для исследования будет использована предложенная в работе [3] модель глазного яблока, в которой упругие свойства задаются тремя упругими константами, из которых две оказываются существенными, характеризуя отдельно упругие свойства роговичной и склеральной областей. Модель [3] достаточно проста и не требует знания множества параметров, определение которых для индивидуального обследуемого глаза в клинике невозможно. Вместе с тем она позволяет учесть неоднородность глазного яблока как упругой системы. Роговица представляется мягкой (безмоментной) двумерной однородной изотропной линейно упругой поверхностью, а склера и прилегающие к ней ткани - упругим элементом, откликающимся изменением объема на изменение давления. Пренебрежение сопротивлением роговицы изгибу обосновано эмпирически и впервые было реализовано в механически корректной постановке в работе [1].

Разработанный в статье [3] метод был эффективно применен к анализу механического смысла данных, получаемых с помощью тонометров, в которых к роговице приложен единственный груз. Форма груза может быть разной - это плоский штамп (тонометр Маклакова) [3], выпуклый штамп (конвекс-тонометр Краснова) [2], вогнутый штамп [8], тонкий стержень [4]. Нагружение глазного яблока в тонометре Шиотца отличается от всех этих ситуаций тем, что в этом тонометре осуществляется комбинированное нагружение вогнутым штампом и стержнем.

\section{ТОНОМЕТР ШИОТЦА: УСТРОЙСТВО И СТАНДАРТНАЯ МЕТОДИКА ИНТЕРПРЕТАЦИИ ДАННЫХ}

Схема нагружения в импрессионном тонометре Шиотца представлена на рис. 1. Роговица лежащего пациента первоначально нагружается так называемой стопой вогнутым грузом с характеристиками, одинаковыми или близкими для всех тонометров рассматриваемого типа: вес стопы равен 11,5 г, а радиус кривизны - 15 мм, т.е. приблизительно вдвое больше, чем у нормальной роговицы человека. В центральной части стопы имеется отверстие, в которое свободно опускается грузик в виде тонкого стержня (плунжера). Диаметр стержня 3 мм, его вдавливающаяся в роговицу поверхность так же, как и стопа, вогнута и имеет тот же радиус кривизны. Вес стержня варьируется. Стандартный вес 5,5 (наиболее употребителен); 7,5; 10; 15 г. 
О внутриглазном давлении судят по глубине погружения стержня под контактную поверхность стопы.

В отличие от тонометра Маклакова, калибровка тонометра Шиотца, т.е. разработка алгоритма пересчета измеряемой геометрической характеристики в давление, невозможна по элементарным формулам даже приблизительно и потребовала разработки специальной теории.

Стандартная обработка данных тонометрии по Шиотцу базируется на двух алгоритмах: сначала по измеренной глубине погружения $h$ и известному весу плунжера $G$ определяется тонометрическое давление $p_{t}$, а затем по $p_{t}$ находится истинное внутриглазное давление $p_{0}$.

Зависимость $p_{t}(h)$ принимается в виде

$$
p_{t}=\frac{G}{\zeta_{1}+\zeta_{2} h}
$$

где $\zeta_{1}$ и $\zeta_{2}$ - раз и навсегда определенные эмпирические константы, $\zeta_{1}=0,107$, $\zeta_{2}=0,0138$. Формула (1) предполагает фиксированные внесистемные единицы измерения: $G$ измеряется в граммах силы; $p_{t}-$ в миллиметрах ртутного столба; $h-$ в единицах Шиотца (1 ед. Шиотца $=50$ мкм). Формула чисто эмпирическая, она основывается на экспериментальных исследованиях, предшествовавших тарировке тонометра Шиотца и показавших, в частности, слабую зависимость функции $p_{t}(G, h)$ от упругих свойств оболочки глаза и линейных размеров, характеризующих глазное яблоко, причем не только для человека, но и для нескольких изученных видов животных [7]. Формулу (1) легко интерпретировать на языке теории размерности: из предположения, что существует связь вида $p_{t}=p_{t}(G, h)$ (с дополнительным присутствием в числе аргументов геометрических параметров - размерности длины и безразмерных), общий вид такой связи $h=f\left(G / p_{t}\right)$, а конкретная форма (1) означает эмпирически установленную линейную зависимость $h$ от величины обратной $p_{t}$. Функция $p_{t}(h)$ в этом случае представляется гиперболой.

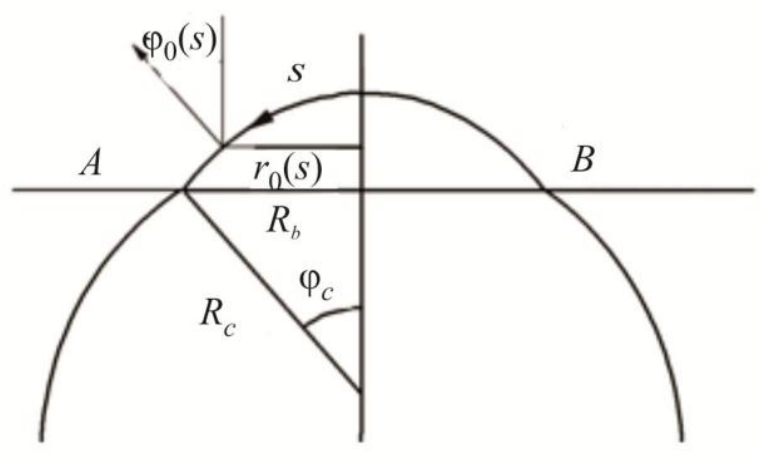

$a$

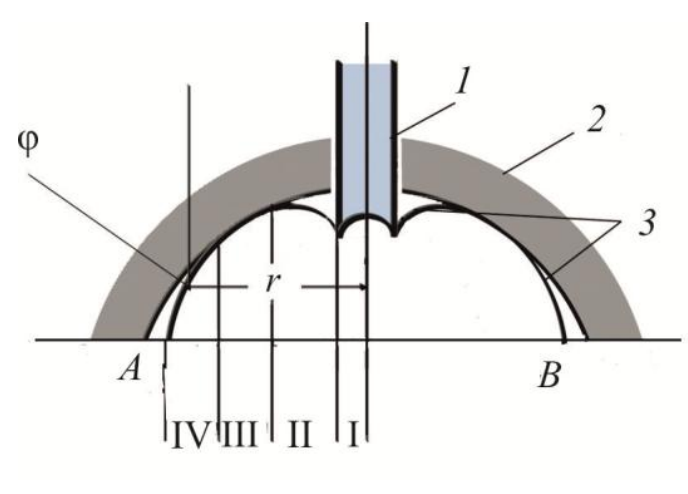

$\sigma$

Рис. 1. К постановке задачи: $a$ - глазное яблоко до нагружения; $\sigma$ - роговица, нагруженная тонометром Шиотца: 1 - плунжер; 2 - стопа; 3 - роговица. Остальные обозначения в тексте 
Расчет $p_{0}$ по $p_{t}$, вычисленному по $h$ в соответствии с формулой (1), базируется на полуэмпирических представлениях, разработанных Фриденвальдом. Для этого вводится так называемый смещенный объем $V_{d}$ - геометрическая характеристика, однозначно (по специальному правилу) определяемая по искривленной поверхности роговицы после наложения груза [6]. Для данного обследуемого глаза (с некоторыми фиксированными геометрическими и механическими свойствами, включая давление до нагружения $\left.p_{0}\right)$ и фиксированного нагружающего устройства связь между $h$ и $V_{d}$ однозначна; поэтому должна существовать связь $p_{t} / p_{0}=\varphi\left(V_{d}\right)$. Эта связь предполагается универсальной (т.е. не зависящей от индивидуальных характеристик глаза) и экспоненциальной по $V_{d}$ :

$$
p_{t} / p_{0}=10^{\alpha V_{d}}
$$

где $\alpha=0,0215$ мм $^{-3}$ - константа Фриденвальда, найденная им как средняя по множеству обследованных глаз.

Неявно считается, что при одинаковых глубине погружения плунжера $h$ и весе плунжера $G$ (а следовательно, с учетом принятой зависимости (1), при одинаковом тонометрическом давлении $p_{t}$ ) геометрия искривленной роговицы одинакова. Отсюда следует однозначная связь $V_{d}\left(p_{t}, G\right)$, которая находится эмпирически и в логарифмических координатах аппроксимируется линейными функциями $\lg V_{d}=\lg V_{d}\left(\lg p_{t}\right)$ с разными коэффициентами для разных весов плунжера. В частности, при $G=5,5$ и 7,5 г результат такой аппроксимации имеет вид $\left(V_{d}\right.$, мм $^{3} ; p_{t}$, мм рт. ст.)

$$
\begin{array}{ll}
G=5,5 \Gamma: & \lg V_{d}=2,016\left(2,029-\lg p_{t}\right), \\
G=7,5 \Gamma: & \lg V_{d}=2,174\left(2,092-\lg p_{t}\right) .
\end{array}
$$

Константы, присутствующие в формуле (3), как и константа Фриденвальда в зависимости (2), найдены как средние для некоторой обширной популяции испытуемых.

Зависимости (1)-(3) непосредственно зашиты в программное обеспечение для используемого в отечественной медицинской практике тонографа GlauTest-60.

\section{ПОСТАНОВКА ЗАДАЧИ И МЕТОД РЕШЕНИЯ}

Общая постановка задачи подробно описана и обоснована в работе [3]. Она включает в себя систему обыкновенных дифференциальных уравнений для описания статических деформаций нагруженной извне оболочки (роговицы), которая дополняется конечным соотношением для склеральной части внутриглазного объема, находящейся вне роговицы (т.е. ниже плоскости $A B$ на рис. 1).

Рассматриваем осесимметричную деформацию поверхности (роговицы), распертой внутренним давлением $p$, причем предполагается, что при некотором давлении $p_{0}$ она имела форму сферического сегмента радиуса $R_{c}$ (рис. $1, a$ ) с радиусом основания $R_{b}$ и углом полураствора $\varphi_{c}\left(\sin \varphi_{c}=R_{b} / R_{c}\right)$. В области свободной роговицы (вне контакта с приложенной нагрузкой) уравнения равновесия и состояния для роговицы как мягкой двумерной упругой поверхности в предположении малых деформаций с учетом конечности смещений имеют вид

$$
\left(r_{0} T_{1}\right)^{\prime}-T_{2} \cos \varphi=0
$$




$$
\begin{gathered}
-T_{2} \sin \varphi-r_{0} \varphi^{\prime} T_{1}+\lambda r_{0} p=0, \\
r^{\prime}=\lambda \cos \varphi, \\
r_{0}=R_{c} \sin \varphi_{0}, \varphi_{0}=\frac{s}{R_{c}}, \lambda=1+\varepsilon_{1}, \varepsilon_{2}=\frac{r}{r_{0}}-1, \\
\varepsilon_{1}=\frac{1}{E_{c}^{*}}\left[T_{1}-v_{c} T_{2}-T_{0}\left(1-v_{c}\right)\right], \varepsilon_{2}=\frac{1}{E_{c}^{*}}\left[T_{2}-v_{c} T_{1}-T_{0}\left(1-v_{c}\right)\right] .
\end{gathered}
$$

Здесь независимым параметром считается длина $s$ дуги образующей в ненагруженном состоянии, отсчитываемая от ее пересечения с осью симметрии $\left(-\varphi_{c} R_{c} \leq s \leq \varphi_{c} R_{c}\right) ; \quad T_{1} \quad$ и $\quad T_{2} \quad-$ касательные усилия в меридиональном и перпендикулярном к нему направлениях; $r$ - расстояние точки поверхности, представляющей роговицу, от оси; $\varphi$ - угол между нормалью к этой поверхности и осью симметрии (рис. $1, \sigma) ; \varepsilon_{1}$ и $\varepsilon_{2}$ - деформации в направлении главных осей; $E_{c}^{*}$ и $v_{c}$ - модуль растяжения и коэффициент Пуассона роговицы как двумерной упругой поверхности [3]. Угол $\varphi$ считается положительным, если нормаль направлена во внешнюю относительно оси сторону. Функции $r_{0}(s)$ и $\varphi_{0}(s)-$ значения функций $r(s)$ и $\varphi(s)$ при начальной сферической конфигурации (см. рис. 1, $a$ ). В этой конфигурации усилия $T_{1}$ и $T_{2}$ равны между собой и $T_{1}=T_{2}=T_{0}=p_{0} R_{c} / 2$.

В случае тонометра Шиотца постановка задачи должна учитывать две области, в которых роговица свободна и ее поведение управляется уравнениями (4), а также две области, в которых она прилегает к жестким поверхностям. Эти четыре области показаны на рис. 1, б. В центральной области I (в окрестности точки $s=0$ ) роговица контактирует с вогнутой поверхностью плунжера, в области III - с вогнутой поверхностью стопы, в областях II и IV роговица свободна. В предположении свободного проскальзывания роговицы по поверхностям контакта система (4) остается справедливой в областях I и III за исключением проекции уравнения равновесия на нормальное к роговице направление (второе уравнение (4)): это соотношение теперь должно включать силу нормальной реакции, действующую со стороны груза. Если не интересоваться распределением силы реакции, уравнение (4) можно не рассматривать и заменить его геометрическими связями: под плунжером (область I)

$$
\sin \varphi=\frac{r}{r_{p}}
$$

где $r_{p}$ - радиус кривизны плунжера, и под стопой (область III)

$$
\sin \varphi=\frac{r}{r_{f}}
$$

где $r_{f}$ - радиус кривизны стопы.

В силу симметрии решение задачи ищется в области $0 \leq s \leq \varphi_{c} R_{c}$. Система уравнений - третьего порядка для свободной роговицы и второго - под плунжером и под стопой. Поскольку три значения $s$, соответствующие границам между областями, заранее неизвестны, требуются 13 дополнительных условий. Эти условия выставляются 
как на внешних границах области решения ( $s=0$ и $s=\varphi_{c} R_{c}$ ), так и на неизвестных внутренних границах. В качестве граничных условий на концах области принимаем (см. [3]) условие $r\left(\varphi_{c} R_{c}\right)=R_{b}$, соответствующее закреплению роговицы на склере, деформации которой считаются малыми, и условие ограниченности усилия $T_{1}$ в точке пересечения с осью симметрии. Последнее принимает форму асимптотического соотношения [3]. Остальные условия формулируются на границах отрыва роговицы от поверхностей. С учетом неразрывности поверхности роговицы, а также условий свободного проскальзывания (сила реакции нормальна к поверхности контакта) и исчезновения силы реакции в точках отрыва получаем следующие условия:

$$
\begin{array}{ll}
\text { I-II: } \quad & r^{-}=r^{+}=r_{*}, T_{1}^{-}=T_{1}^{+}, \\
& G=\pi r_{*}^{2} \cdot p-T_{1} \sin \left(-\varphi^{-}\right) \cdot 2 \pi r_{*} \\
\text { II-III: } & r^{-}=r^{+}, \quad \varphi^{-}=\varphi^{+}, T_{1}^{-}=T_{1}^{+} ; \\
& r^{-}=r^{+}, \varphi^{-}=\varphi^{+}, T_{1}^{-}=T_{1}^{+}, \\
\text {III-IV: } & G+G_{f}=\pi r^{2} \cdot p-T_{1} \sin \varphi \cdot 2 \pi r .
\end{array}
$$

Здесь $G$ и $G_{f}-$ вес стержня и стопы соответственно; $r_{*}$ - радиус плунжера, а индексами «-» и «+» обозначены значения переменных по разные стороны линии отрыва, причем «-» соответствует области, лежащей от оси дальше. В соотношениях, содержащих вес (условиях равновесия), функции, непрерывные на рассматриваемой поверхности разрыва, индексами «-» и «+» не помечены. Каждая из систем соотношений (7)-(9) соответствует границе между указанными областями. Граничные условия (7) сформулированы ранее в работе [4] при решении задачи о чисто стержневом нагружении роговицы.

Система уравнений (4)-(5) с указанными граничными условиями дает возможность вычислять геометрические характеристики деформированной под грузом роговицы по известным $p, G$ и $G_{f}$. В числе таких характеристик приращение $\Delta V_{c}$ подроговичного объема (часть внутриглазного объема над плоскостью $A B$ ), а также расстояния от точек деформированной роговицы до плоскости $A B$, необходимые для расчета измеряемого погружения плунжера. Таким образом, в силу постановки задачи геометрия деформированной роговицы определяется, помимо внутреннего давления, только геометрическими и механическими характеристиками самой роговицы. Чтобы связать $\Delta V_{c}$ с приращением полного заполненного жидкостью внутриглазного объема $\Delta V$, вводится склеральный объем (под $A B$ ) с приращением $\Delta V_{s}$, подчиняющимся соотношениям

$$
\Delta V_{s}=K\left(p-p_{0}\right), \quad \Delta V=\Delta V_{c}+\Delta V_{s},
$$

где $K$ - интегральная константа, определяющая упругое поведение всей склеральной области и зависящая не только от механических, но и от геометрических характеристик, в частности от ее объема. При сохранении механических свойств склеры и ее окружения больший объем внутрисклерального пространства (например, при миопии) приведет к увеличению $K$, т.е. к уменьшению эффективной жесткости склеральной области.

В рамках модели свойства объекта (глазного яблока) определяются пятью параметрами. В их числе три, характеризующие его упругие свойства (но включающие в 
себя зависимость и от геометрических характеристик): $E_{c}^{*}, v_{c}$ и $K$. Два других - чисто геометрические, характеризующие форму роговицы: ее радиус $R_{c}$ и радиус основания роговицы $R_{b}$ ). Как продемонстрировано в работе [3], вместо линейной жесткости роговицы $E_{c}^{*}$ удобно ввести ее поверхностную жесткость $E_{c s}^{*}=E_{c}^{*} /\left(1-v_{c}\right)$. В задачах, где главную роль играет изотропное растяжение роговицы (а именно таковы задачи тонометрии), влияние коэффициента Пуассона $v_{c}$ отдельно от $E_{c s}^{*}$ несущественно.

Связь этих констант с традиционно используемыми обсуждалась ранее [3]. Для удобства сравнения с данными других авторов были формально определены роговичная и склеральная жесткости $E_{c}$ и $E_{s}$, измеряемые в мегапаскалях и отличающиеся от $E_{c}^{*}$ и $K^{-1}$ фиксированными размерными множителями: $E_{c}^{*}=\delta E_{c}$, $K^{-1}=\zeta E_{s}$, где $\delta=0,5 \mathrm{Mм}-$ средняя в норме толщина роговицы, а $\zeta=1,44 \cdot 10^{-5} \mathrm{Mм}^{-3}$. Эти величины имеют простой физический смысл в рамках сильно упрощенных моделей (см., например, [3]). Расчеты в основном проводились при стандартных для человеческого глаза значениях $R_{c}=8 \mathrm{~mm}, R_{b}=5,8$ мм. Изменение коэффициента Пуассона роговицы приводит лишь к переопределению $E_{c}$, по той же причине (удобство сравнения с другими данными) он выбирался равным 0,45 , что соответствует в случае трехмерной изотропной оболочки почти несжимаемому материалу. Выполнялись еще расчеты при других значениях геометрических параметров: $R_{c}=7 \ldots 9$ мм, $R_{b}=5,4 \ldots 6,2$ мм, а также при коэффициентах Пуассона $\mathrm{v}_{c}=0,25 \ldots 0,65$. В последнем случае сравнение проводилось с расчетами для измененного значения линейного модуля $E_{c}^{\prime}=E_{c}\left(1-v_{c}\right) /(1-0,45)$, т.е. при том же поверхностном упругом модуле $E_{c s}=E_{c} /\left(1-v_{c}\right)$. Подтверждена слабая зависимость результатов от коэффициента Пуассона при таком выборе параметров.

В работе [3] оценены «средние» по множеству относительно здоровых (неглаукомных) глаз значения упругих констант: $E_{s}=10$ МПа, $E_{c}=0,3$ МПа. Всюду далее, когда говорится о средних (нормальных) значениях, имеются в виду именно вышеуказанные. С учетом высокого индивидуального (в том числе вследствие патологий) разброса влияние этих констант исследовалось в диапазонах $E_{s}=2 \ldots 20 \mathrm{M \Pi а}, E_{c}=0,1 \ldots 0,7$ МПа.

Единственный параметр нагружающего устройства, который варьировался, - вес плунжера $G$. Расчеты проводились для $G=5,5$ и 7,5 г. Остальные параметры тонометра, как и в медицинской практике, выбирались следующими: радиус контактной поверхности стопы $r_{f}=15$ мм, радиус вогнутого основания плунжера $r_{p}=r_{f}=15$ мм, вес стопы $G_{f}=11,5$ г.

В основе рассмотрения было изучение функции полного объема $\Delta V=\Delta V\left(p, G ; E_{c}, E_{s}\right)$, состоявшее из трех этапов: определение $\Delta V_{c}$ из решения краевой задачи для роговицы, а затем $\Delta V_{s}$ и $\Delta V$ по формулам (10). Формально в числе аргументов функции $\Delta V$ должно присутствовать давление $p_{0}$, соответствующее условно выбранной начальной сферической конфигурации, однако его влияние на исследуемые зависимости мало [6]; поэтому этот аргумент явно не указан. При численном решении применялись метод стрельбы и схема Рунге-Кутты четвертого порядка. 


\section{СВЯЗЬ ГЛУБИНЫ ПОГРУЖЕНИЯ ПЛУНЖЕРА С ТОНОМЕТРИЧЕСКИМ ДАВЛЕНИЕМ}

В случае тонометра Шиотца измеряемая геометрическая характеристика глубина погружения плунжера $h$, отсчитываемая от внутренней поверхности стопы. При стандартной обработке по формуле (1) считается, что на связь ее с тонометрическим давлением влияет только вес плунжера.

В рамках используемой модели тонометрическое давление есть функция не только $G, h$ и геометрических параметров роговицы $R_{c}$ и $R_{b}$, но и упругости роговицы, а также веса стопы: $p_{t}=p_{t}\left(G, h, R_{c}, R_{b}, E_{c}, G_{f}\right)$. Зависимость от склеральной жесткости отсутствует в силу постановки задачи для роговицы (в которой деформация склеры не учитывается). Поскольку вес стопы в тонометре Шиотца всегда одинаков, далее исследуется влияние на результат восстановления значения тонометрического давления по величине $h$ только параметров $E_{c}, R_{c}$ и $R_{b}$.

На рис. 2 представлена зависимость тонометрического давления $p_{t}$ от глубины погружения плунжера $h$ для различных жесткостей роговицы $E_{c}$ и двух стандартных весов плунжера в сравнении с эмпирической формулой (1). Рассчитанные кривые подтверждают как весьма слабую зависимость кривых $p_{t}(h)$ от $E_{c}$, так и пригодность в первом приближении формулы (1). Во всем практически важном диапазоне тонометрическое давление, соответствующее данной фиксированной $h$, меньше, чем определяемое формулой (1) (кроме давлений ниже 20 мм рт. ст.) и растет с ростом жесткости роговицы. В области низких тонометрических давлений (ниже 25 мм рт. ст.) совпадение всех кривых (при разных $E_{c}$ и эмпирической) практически полное, однако с ростом давления отклонение нарастает и для $G=5,5$ г при $p_{t} \sim 30$ мм рт. ст. имеет порядок 3 мм рт. ст., а для $G=7,5$ г при $p_{t} \sim 35 \ldots 50$ мм порядок 5 мм рт. ст. Для груза $G=5,5$ г с ростом $p_{t}$ выше 30 мм рт. ст. наблюдается сокращение разницы результатов, рассчитанных по модели и полученных по эмпирической формуле, и при 40-50 мм рт. ст. они вновь практически совпадают.

Влияние радиуса роговицы на зависимость тонометрического давления от заглубления представлено на рис. 3 , где приведены результаты расчетов зависимостей $p_{t}(h)$ при разных радиусах роговицы $R_{c}$ для $G=5,5$ г и $E_{c}=0,3$ МПа. Эта величина может отличаться от стандартного для человека значения в результате операций на роговице. Также она иная и у детей. Возрастание радиуса кривизны роговицы приводит к уменьшению реального тонометрического давления при той же $h$. Таким образом, при увеличенном радиусе кривизны использование зависимостей, полученных при стандартном значении радиуса $R_{c}$, дает завышенный результат, что указывает на возможность ошибок измерения на прооперированных глазах. Это расхождение особенно заметно при достаточно малых $h$, когда возможна ошибка до 3 мм рт. ст. Изменение радиуса кривизны роговицы на 1 мм практически не сказывается на кривых $p_{t}(h)$ при $p_{t} \sim 35$ мм рт. ст. и выше и достигает 2-3 мм рт. ст. при $p_{t} \sim 20$ мм рт. ст.

Вычисление зависимостей $p_{t}(h)$ при разных радиусах основания роговицы $R_{b}=5,4 \ldots 6,2$ мм продемонстрировало практически полное совпадение соответствующих кривых. Таким образом, влияние параметра $R_{b}$ на вид зависимости $p_{t}(h)$ можно не учитывать. 


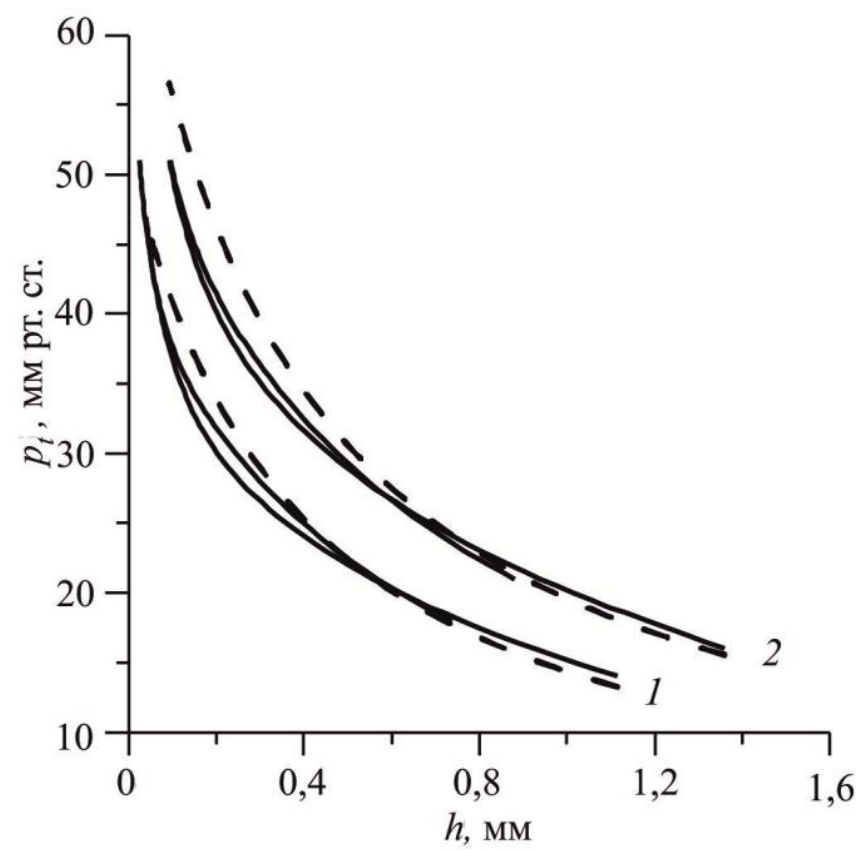

Рис. 2. Зависимость тонометрического давления $p_{t}$ от веса плунжера и жесткости роговицы. Семейство кривых 1 соответствует весу плунжера $G=5,5$ г, семейство $2-$ весу $G=7,5$ г. Сплошные кривые: нижняя в каждом семействе $-E_{c}=0,2$ МПа, верхняя $-E_{c}=0,6$ МПа; пунктирные - рассчитанные по стандартной формуле (1) для соответствующего веса $G$

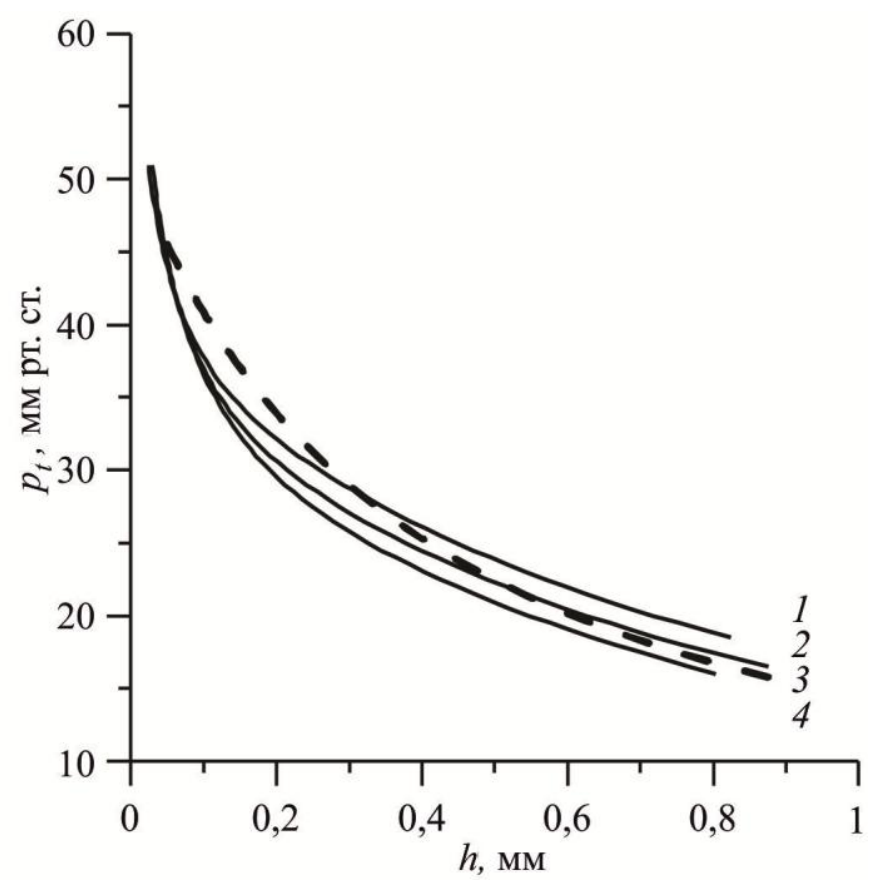

Рис. 3. Зависимость тонометрического давления $p_{t}$ от радиуса кривизны роговицы $R_{c}$ при весе плунжера $G=5,5$ г и жесткости роговицы $E_{c}=0,3$ МПа: 1 $R_{c}=7$ мм; $2-R_{c}=8$ мм; $4-R_{c}=9$ мм; пунктирная кривая 3 построена по стандартной формуле (1) 
В целом расчеты показывают, что формула (1) вполне пригодна для оценки тонометрического давления, но в некоторых случаях все же дает заметные отклонения от реальных значений $p_{t}$. Эти отклонения наиболее заметны на интервале умеренно больших $p_{t}$ (разном для разных весов плунжера) и увеличиваются при возрастании веса плунжера и радиуса кривизны роговицы.

\section{ОПРЕДЕЛЕНИЕ ИСТИННОГО ДАВЛЕНИЯ ПО ТОНОМЕТРИЧЕСКОМУ}

Задача тонометрии решалась численно подбором такого значения давления $p_{0}$, при котором приложение нагрузки не меняет объем: $\Delta V=0$. В этом случае $p_{0}$ соответствует внутриглазному давлению до нагружения. Целью исследования было изучить влияние индивидуальных механических и геометрических характеристик глаза на зависимость между $p_{0}$ и $p_{t}$ (тонометрическую зависимость) и оценить область приложимости формул (2), (3).

На рис. 4, 5 приведены результаты расчетов тонометрических зависимостей при различных роговичной и склеральной жесткостях для $G=5,5$ г. Представлены зависимость истинного давления $p_{0}$ от тонометрического $p_{t}$ (см. рис. 4) и тонометрической разности $\Delta p=p_{t}-p_{0}$ от $p_{0}$ (см. рис. 5). Тонометрическая разность зависит как от уровня давления, так и от обеих жесткостей. С ростом давления эта величина уменьшается (см. рис. 5).

Увеличение склеральной жесткости $E_{s}$ (рис. 4, a) приводит к уменьшению истинного давления $p_{0}$, соответствующего измеренному тонометрическому давлению $p_{t}$. Различие значительно: при изменении $E_{s}$ от 6 до 20 МПа ( $E_{c}=0,3$ МПа) оно достигает 6 мм рт. ст. Практически важно, что $E_{s}$ слабо сказывается на форме тонометрических зависимостей: кривые $p_{0}\left(p_{t}\right)$ при разных $E_{s}$ визуально подобны и различаются лишь сдвигом по оси ординат.

Совсем по-иному сказывается жесткость роговицы $E_{c}$ (рис. 4, б). Возрастание этой жесткости приводит уже к возрастанию $p_{0}$, соответствующего некоторому измеренному $p_{t}$. Как функция от $p_{0}$ тонометрическая разность $\Delta p$ и в этом случае убывает, но существенно неравномерно для разных $p_{0}$ : при $E_{s}=10$ МПа изменение $E_{c}$ от 0,2 до 0,7 МПа практически не влияет на $\Delta p$ при $p_{0}=10$ мм рт. ст. (различие не более 0,3 мм рт. ст.), но с возрастанием $p_{0}$ это влияние становится все более заметно, и при $p_{0}=30$ мм рт. ст. различие достигает 3,5 мм рт. ст. Визуально это проявляется в том, что семейство кривых $p_{0}\left(p_{t}\right)$ при разных $E_{c}$ расходится веером, расширяющимся в сторону возрастания давлений (см. рис. $4, \sigma)$.

Результаты расчета связи $p_{0}\left(p_{t}\right)$ по нашей модели при средних значениях упругих констант, т.е. близких к $E_{s}=10 \mathrm{MПа,} E_{s}=0,3$ МПа, и весе плунжера $G=5,5$ г практически совпадают с результатом применения стандартных формул (2), (3) (рис. 6). Однако совпадение имеет место лишь для нормальных глаз: при повышенной жесткости роговицы стандартный расчет по тонометрическому давлению занижает, а при повышенной склеральной жесткости завышает истинное давление. 


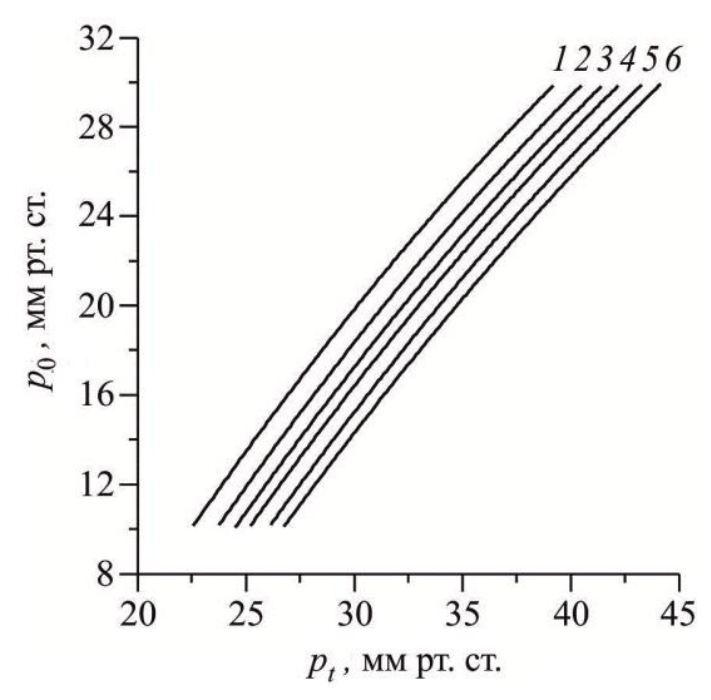

$a$

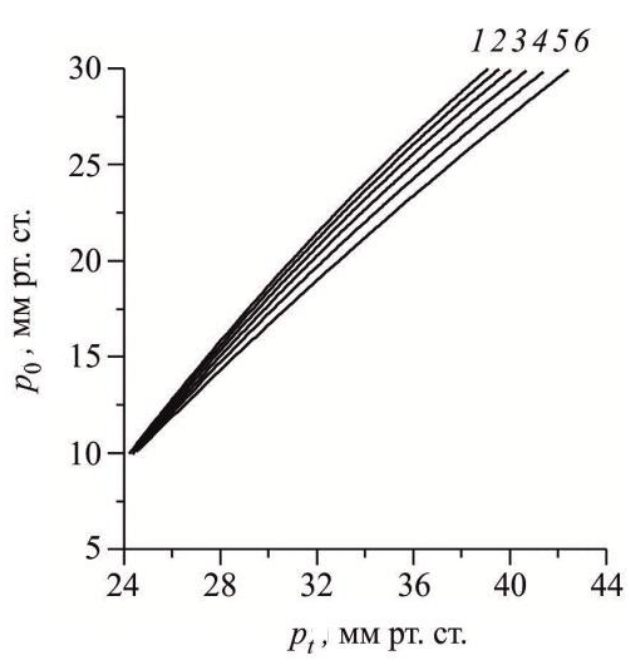

$\sigma$

Рис. 4. Влияние упругих свойств роговицы и склерального сегмента на зависимость истинного давления $p_{0}$ от тонометрического $p_{t}: a-E_{c}=0,3 \mathrm{MПа,} E_{s}=6$ МПа (кривая 1), $E_{s}=8$ МПа (кривая 2), $E_{s}=10$ МПа (кривая 3), $E_{s}=12$ МПа (кривая 4), $E_{s}=16$ МПа (кривая 5), $E_{s}=20$ МПа (кривая 6); $\sigma-E_{s}=10$ МПа, $E_{c}=0,7$ МПа (кривая 1), $E_{c}=0,6$ МПа (кривая 2), $E_{c}=0,5$ МПа (кривая 3), $E_{c}=0,4$ МПа (кривая 4), $E_{c}=0,3$ МПа (кривая 5), $E_{c}=0,2$ МПа (кривая 6)

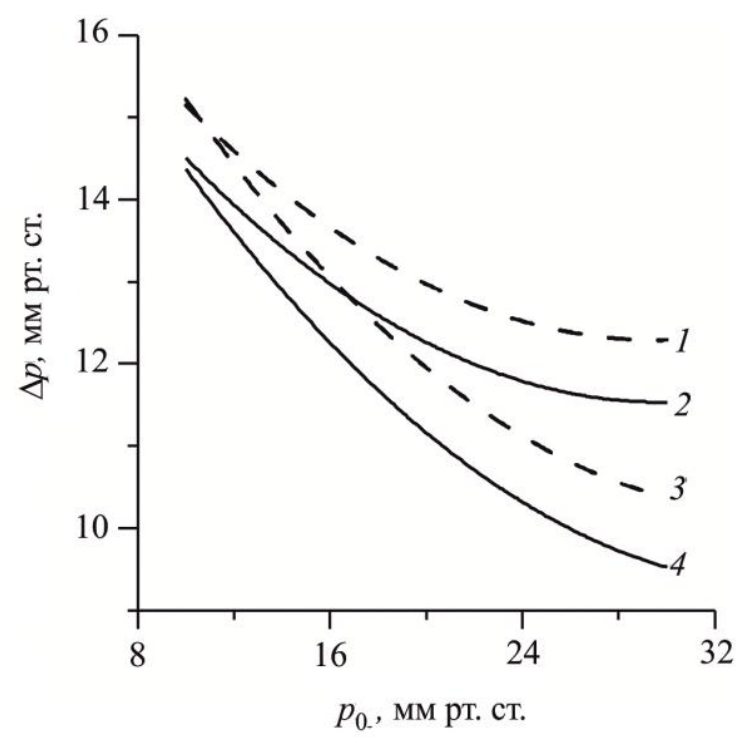

Рис. 5. Влияние упругих свойств роговицы и склерального сегмента на зависимость тонометрической разности $\Delta p$ от истинного давления $p_{0}:$ кривая 1 $E_{c}=0,3$ МПа, $E_{s}=12$ МПа; $2-E_{c}=0,3$ МПа, $E_{s}=10$ МПа; $3-E_{c}=0,3$ МПа, $E_{s}=12$ МПа; $4-E_{c}=0,3$ МПа, $E_{s}=10$ МПа

При весе плунжера $G=7,5$ г расчетная кривая $p_{0}\left(p_{t}\right)$ дает при нормальных упругих константах значения истинного давления приблизительно на 3 мм рт. ст. выше, чем стандартная методика. При значениях упругих констант, отличных от нормальных, наблюдаются те же закономерности, что и при $G=5,5$ г. Таким образом, для $G=7,5$ г 


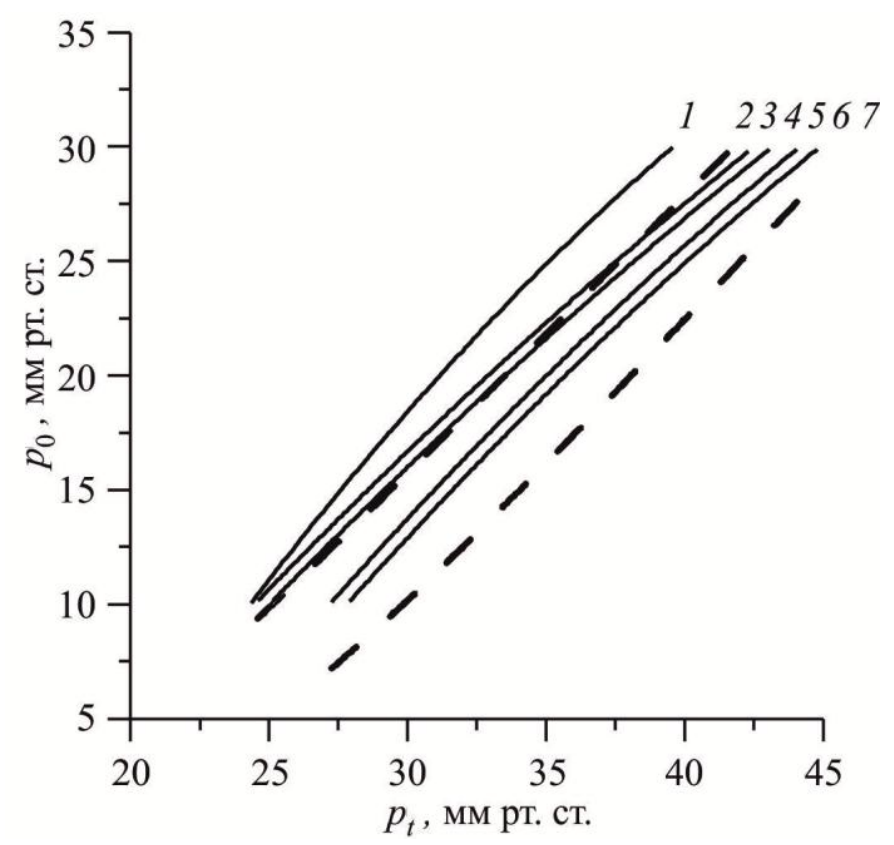

Рис. 6. Сопоставление зависимостей истинного давления $p_{0}$ от тонометрического $p_{t}$, рассчитанных по принятой модели, с зависимостями, получаемыми по стандартным формулам (2), (3) при разных весах плунжера. Кривые 1-4 соответствуют весу $G=5,5$ г: $1-E_{c}=0,6$ МПа, $E_{s}=10$ МПа; 2 - расчет по формулам (2), (3); $3-E_{c}=0,2 \mathrm{MПа,} E_{s}=10 \mathrm{MПа;} 4-E_{c}=0,2 \mathrm{MПа}$, $E_{s}=12$ МПа; кривые 5-7 соответствуют весу $G=7,5$ г: $5-E_{c}=0,2$ МПа, $E_{s}=10 \mathrm{MПа;} 6-E_{c}=0,2 \mathrm{MПа,} E_{s}=12 \mathrm{MПа;} \mathrm{7-} \mathrm{расчет} \mathrm{по} \mathrm{формулам} \mathrm{(2),} \mathrm{(3)}$

повышенная жесткость роговицы и (или) пониженная склеральная жесткость увеличивают отклонение от результатов стандартного расчета, а повышенная склеральная жесткость его уменьшает.

Тонометрические кривые претерпевают некоторые изменения при изменении геометрических параметров роговицы (форма и размеры склеры учитываются условной склеральной жесткостью $\left.E_{s}\right)$. На рис. 7 представлена зависимость $p_{0}\left(p_{t}\right)$ для разных радиусов кривизны роговицы $R_{c}$ при средних значениях упругих параметров. Увеличение радиуса кривизны приводит к некоторому повышению $p_{0}$, соответствующего тому же $p_{t}$. При малых давлениях ( $p_{0} \sim 10$ мм рт. ст.) изменение $R_{c}$ на 1 мм соответствует изменению $p_{0}$ порядка 2 мм рт. ст. С увеличением $p_{0}$ (или $p_{t}$ ) влияние $R_{c}$ ослабевает и при $p_{0}>20$ мм рт. ст. практически пропадает.

Влияние радиуса основания роговицы $R_{b}$ более равномерно (рис. 8): при возрастании $R_{b}$ кривые $p_{0}\left(p_{t}\right)$ остаются визуально подобны, сдвигаясь кверху вдоль оси ординат. При увеличении $R_{b}$ на 0,8 мм от 5,4 до 6,2 мм давление $p_{0}$, соответствующее заданному $p_{t}$, возрастает на величину порядка 2 мм рт. ст. Следует, впрочем, иметь в виду, что радиус $R_{b}$ у человека очень стабилен. Различия при его определении связаны не с индивидуальным разбросом, а с анатомической привязкой границы роговицы. В рамках рассматриваемого огрубленного моделирования выбор значения $R_{b}$ в известной мере условен и его небольшое изменение фактически сведется к некоторому переопределению упругих констант. 


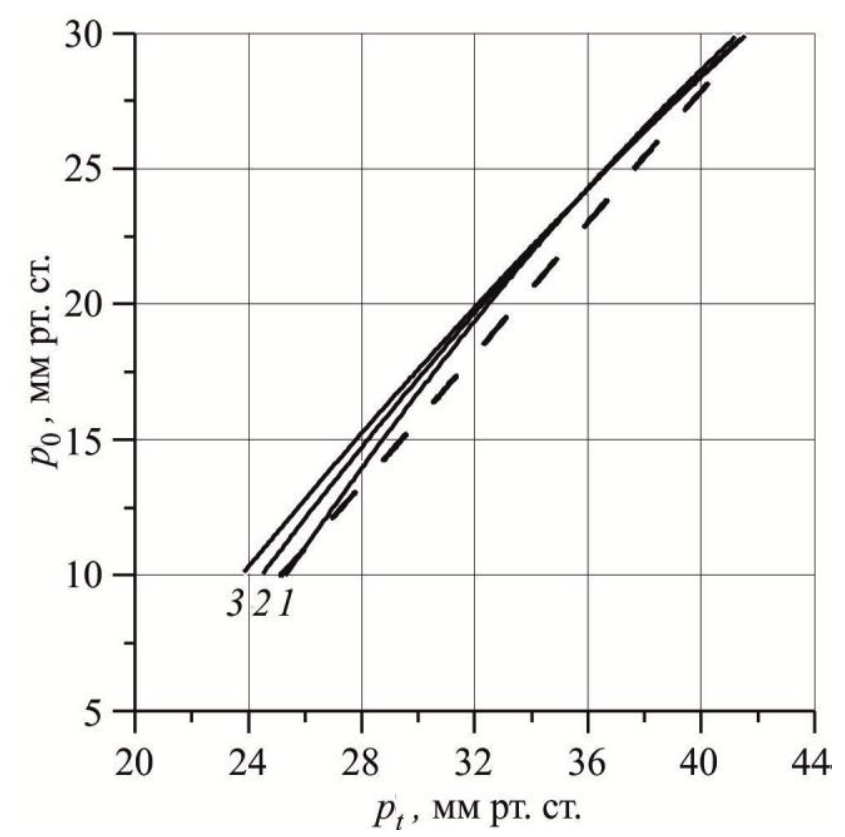

Рис. 7. Влияние радиуса кривизны роговицы на зависимость истинного давления $p_{0}$ от тонометрического $p_{t}$ при $G=5,5 \Gamma, E_{c}=0,3$ МПа, $E_{s}=10$ МПа. Сплошные кривые: $1-R_{c}=7 ; 2-R_{c}=8 ; 3-R_{c}=9$ мм; пунктирная кривая - расчет по стандартным формулам (2), (3)

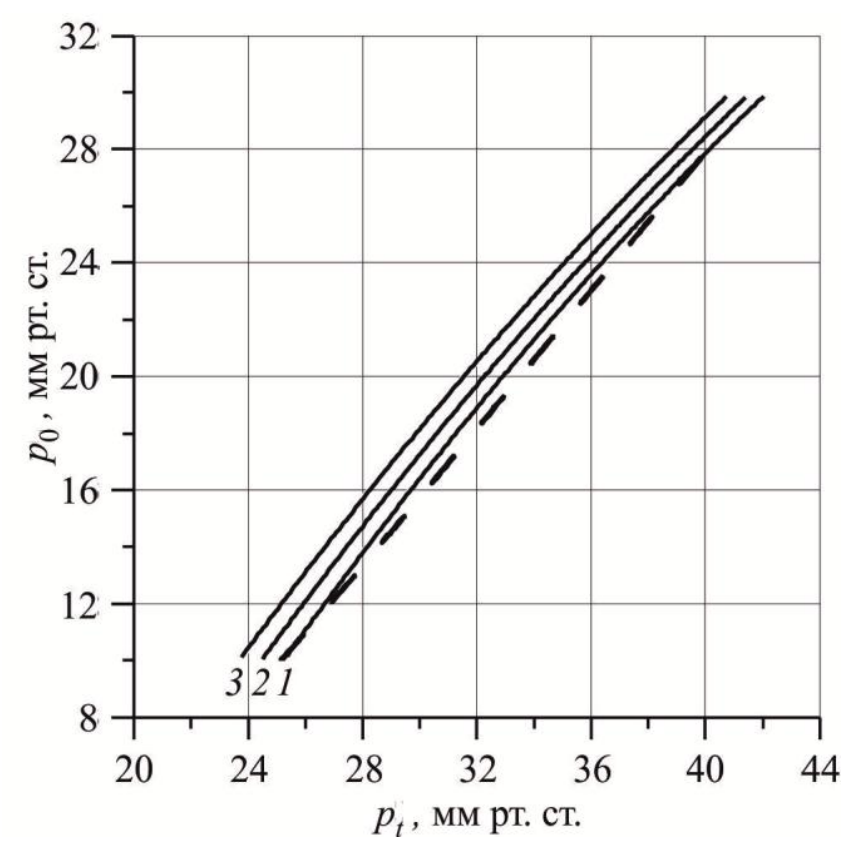

Рис. 8. Влияние радиуса основания роговицы на зависимость истинного давления $p_{0}$ от тонометрического $p_{t}$ при $G=5,5$ г, $E_{c}=0,3$ МПа, $E_{s}=10$ МПа. Сплошные кривые: $1-R_{b}=5,4 ; 2-R_{b}=5,8 ; 3-R_{b}=6,2$ мм; пунктирная кривая - расчет по стандартным формулам (2), (3)

Таким образом, формулы, используемые при стандартной обработке данных, вполне применимы, если упругие характеристики глазного яблока близки к средним. При наличии существенных отклонений этих величин от средних значений оценка по 
стандартным формулам может давать существенную ошибку. Кроме того, ошибка возрастает для плунжера большего веса. Возможно, применение большего веса плунжера правомерно для глаз с большой склеральной жесткостью.

\section{Выводы}

Разработанная авторами ранее простая механическая модель глазного яблока позволила механически корректно исследовать вопрос о применимости стандартных формул, используемых при обработке данных, получаемых при тонометрии по Шиотцу. Расчеты показали, что оценка тонометрического давления для тонометров с весами плунжера 5,5 и 7,5 г по стандартной методике в целом правомерна, хотя в некоторых диапазонах давлений ошибка может и увеличиваться. Оценка истинного давления для тонометра с минимальным весом плунжера 5,5 г по стандартным формулам соответствует расчетным данным при средних значениях упругих констант, но заметно отклоняется от них, если эти константы сильно отличаются от средних значений. При большем весе плунжера $(7,5$ г) расчетные кривые заметнее отличаются от полученных по стандартной методике даже и для средних значений констант. Влияние геометрических параметров роговицы не слишком велико, однако при сильно увеличенном радиусе ее кривизны (например, в результате операции) поправки к результатам стандартного расчета следует учитывать.

Вопрос о диагностической ценности различных оцениваемых при тонометрии параметров (глубины погружения плунжера, истинного и тонометрического давления и т.д.) должен служить предметом медицинских исследований.

\section{БЛАГОДАРНОСТИ}

Авторы благодарны Г.А. Любимову за ценные замечания. Работа выполнена при поддержке гранта РФФИ № 14_01_00475.

\section{СПИСОК ЛИТЕРАТУРЫ}

1. Бауэр С.М., Любимов Г.А., Товстик П.Е. Математическое моделирование метода Маклакова измерения внутриглазного давления // Изв. РАН. Мех. жидк. и газа. - 2005. - № 1. - С. 24-39.

2. Любимов Г.А., Моисеева И.Н., Штейн А.А. Исследование свойств двухкомпонентной механической модели глазного яблока и возможности ее использования при практической оценке механических свойств глаза человека // Изв. РАН. Мех. жидк. и газа. - 2014. - № 6. - С. 5-16.

3. Моисеева И.Н., Штейн А.А. Анализ зависимости давление - объем для глазного яблока, нагруженного плоским штампом, на основе двухсегментной упругой модели // Изв. РАН. Мех. жидк. и газа. -2011 . - № 5. - С. 3-15.

4. Моисеева И.Н., Штейн А.А. Исследование зависимости давление - объем для глазного яблока при нагружении тонким стержнем // Изв. РАН. Мех. жидк. и газа. - 2013. - № 4. - С. 3-14.

5. Нестеров А.П., Бунин А.Я., Кацнельсон Л.А. Внутриглазное давление. Физиология и патология. - М.: Наука, 1974. - $381 \mathrm{c}$.

6. Friedenwald J.S. Contribution to the theory and practice of tonometry // Am. J. Ophthalmol. - 1937. Vol. 20, № 10. - P. 985-1024.

7. Friedenwald J.S. Some problems in the calibration of tonometers // Am. J. Ophthalmol. - 1948. - Vol. 31. - P. 935-944.

8. Friedenwald J.S. Standartization of tonometers; decennial report by the committee on standartization of tonometers. - Omaha: Am. Acad. Ophthalmol., 1954. - 177 c.

9. Moses R.A. Theory of the Schiøtz tonometer and its empirical calibration // Trans. Am. Ophthalmol. Soc. 1971. - Vol. 69. - P. 494-562.

10. Pallikaris I.G., Kymionis G.D., Ginis H.S., Kounis G.A., Tsilimbaris M.K. Ocular rigidity in living human eyes // Invest. Ophthalm. Vis. Sci. - 2005. - Vol. 46, № 2. - P. 409-414. 


\section{ESTIMATING THE CORRECTNESS OF THE STANDARD METHOD USED IN CALCULATING THE INTRAOCULAR PRESSURE FROM SCHIØTZ TONOMETRY}

\section{I.N. Moiseeva, A.A. Stein (Moscow, Russia)}

The dependences used in the standard processing of the Schiøtz tonometry data for estimating the intraocular pressure are analyzed. In the Schiøtz tonometer, the eye cornea is loaded together by a heavy concave footplate and a thin lighter concave rod (plunger). The loading device contacts the cornea in two zones: the entire concave rod surface and a footplate region determined by the internal pressure. The mathematical modelling uses the model proposed by the authors earlier, in which the cornea is represented by a momentless (soft) shell and the scleral region is modeled by an elastic element that responses to changes in pressure by changes in volume. The standard processing of the Schiøtz tonometry data is based on two algorithms justified empirically: first, from the measured depth of plunger indentation under the footplate and the known plunger weight the tonometric pressure is determined and then, from the latter the intraocular pressure in the unloaded eye ("true" pressure) is calculated. It is shown that the standard method is quite acceptable for the tonometer with a minimum rod weight of $5.5 \mathrm{~g}$ if the elastic characteristics of the eyeball are close to average values. For a greater rod weight or elastic constants substantially different from the normal values, it is necessary to take corrections into account. The influence of the geometric parameters of the cornea is moderate but for corneas with strongly increased radius of curvature, for example, owing to surgery, corrections to the standard calculation results should be taken into account.

Key words: eye, mathematical models, eye shell, intraocular pressure, tonometry, Schiøtz tonometer. 